\title{
Clinical value and application of contrast-enhanced ultrasound in the differential diagnosis of malignant and benign breast lesions
}

\author{
YAN ZHANG, BMEIWU ZHANG, XIAOXIANG FAN and DAFENG MAO \\ Department of Interventional Therapy, Ningbo No. 2 Hospital, Ningbo, Zhejiang 315010, P.R. China
}

Received March 12, 2018; Accepted July 29, 2019

DOI: $10.3892 /$ etm.2020.8895

\begin{abstract}
The aim of the present study was to assess the performance of contrast-enhanced ultrasound in distinguishing between malignant and benign breast lesions and the diagnostic value of its clinical application. A total of 52 cases with malignant breast tumors and 73 cases with benign breast lesions were included in the study. Time-intensity curves (TICs) for contrast-enhanced ultrasound were recorded, and the perfusion parameters were obtained and analyzed. Typical features of malignant breast tumors included irregular shape and vascular morphology, uneven contrast agent distribution, filling defects and contrast agent retention, 'fast-out' wash-out mode, unclear boundaries and uneven internal echo. Benign lesions were characterized by 'slow-out' or synchronous wash-out mode. Regarding perfusion, the starting time of the perfusion of the Sone-Vue microbubble contrast (always 20-30 sec) and time to peak (TTP) were significantly earlier for the malignant lesions, while the wash-out time was later. A significantly greater peak intensity, rising slope and area under the TIC were observed for the malignant breast lesions. All of the malignant breast lesions exhibited an enlarged focus scope on ultrasound, while no obvious focus scope enhancement was observed for benign breast lesions. Furthermore, the TICs of $88.4 \%$ of malignant breast lesions were of the fast-rising and slow-declining type, while the TICs of 75.3 and $17.8 \%$ of the benign breast lesions were of the slow-rising and fastdeclining, and fast-rising and fast-declining type, respectively. Receiver operating characteristics analysis indicated that the TTP, wash-out time and rising slope might contribute to the differential diagnosis between malignant and benign breast lesions. In conclusion, TIC parameters of contrast-enhanced ultrasound have promising clinical value in differentiating between malignant and benign breast lesions. The TTP, wash-
\end{abstract}

Correspondence to: Dr Yan Zhang, Department of Interventional Therapy, Ningbo No. 2 Hospital, 41 West North Street, Haishu District, Ningbo, Zhejiang 315010, P.R. China

E-mail: Zhangynb2@tom.com

Key words: contrast-enhanced ultrasound, malignant and benign breast lesions, time-intensity curve, receiver operating characteristic curve out time and rising slope may contribute to the diagnosis of patients with breast lesions to facilitate timely treatment and prognostication of breast cancer patients.

\section{Introduction}

Breast cancer is a common disease that frequently occurs in females. With the rapid changes in lifestyle, the incidence of breast cancer has been increasing year by year (1), particularly in young women (typically <30 years; ranging between 12-65 years in this present study) (2). Due to the atypical manifestations of breast cancer and the fact that breast cancer with a diameter of $<2 \mathrm{~cm}$ is relatively difficult to identify by palpation, the disease is associated with relatively high mortality (3). Therefore, the early detection and treatment of breast cancer are of great importance to reduce the incidence and associated mortality (4). The imaging performance of breast cancer, particularly for small tumors, is complex and diverse, leading to ambiguous diagnostic imaging results (5). Ultrasound detection is characterized by its fast, convenient, non-invasive, low-cost and repeatable clinical application; it has been widely accepted as the preferred screening method for breast cancer and has an irreplaceable role in its diagnosis (6).

Mammography has become a current research hotspot in the forefront of the ultrasound field. With the help of the ultrasonic contrast agent SonoVue tracer and post-processing technology, real-time dynamic tracking of contrast provides information on the features of the tumor tissue and blood vessel perfusion mode, including the time-intensity curve (TIC) (7), perfusion process, washout features and overall distribution, which reflect differences in the tumor vessel-associated perfusion time sequence and their spatial distribution. Through a receiver operating characteristic (ROC) curve analysis, parameters from the TIC have been indicated to enhance the diagnostic performance (8).

In recent years, with the rapid development of contrastenhanced ultrasound technology, its clinical value in the differential diagnosis of liver cancer has been widely recognized (9). However, its application in diagnosing breast diseases remains under investigation, and only few studies have assessed ultrasound imaging parameters in the diagnosis of associated diseases. In order to improve the differential diagnosis of benign and malignant breast tumors, the perfusion characteristics of the real-time contrast-enhanced ultrasound of breast masses were investigated in the present study. 


\section{Materials and methods}

Study subjects. A total of 125 female subjects were included in the present study, with a mean age of $42.6 \pm 17.3$ years, median age of 34.5 years (range, 12-65 years), who underwent ultrasound angiography at Ningbo No. 2 Hospital (Ningbo, China) between August 2015 and August 2017. All of these patients had a breast mass (single lesion) detected on ultrasound, of which 52 cases had malignant tumors and 73 had benign lesions, as confirmed by percutaneous biopsy and postoperative pathology. According to the inclusion criteria, lesion imaging features were selected, and the differential examination for benign and malignant lesions mainly focused on the early diagnosis. The mass diameter ranged from 9 to $45 \mathrm{~mm}$. The disease history was carefully checked prior to examination. Prior written informed consent was obtained from each patient and the study was approved by the Ethics Review Board of the Ningbo No. 2 Hospital (Ningbo, Zhejiang, China).

Real-time contrast-enhanced ultrasound detection. Realtime contrast-enhanced ultrasound was imaging performed with the DU8 Color Doppler Ultrasonic diagnostic instrument (EsaoteSpA), equipped with the superficial conventional linear array probe LA532 with the frequency of $4-13 \mathrm{MHz}$, as well as the contrast probe LA532E with the frequency of 4.5-7.5 MHz. SonoVue microbubble contrast agent (Bracco SpA) was used for detection. Patients were placed in the supine position. The morphological boundary of the lesion was detected in the two-dimensional mode and the blood flow distribution within the lesion was observed using the color and energy Doppler mode. Intravenous access was established and $2.4 \mathrm{ml}$ SonoVue was rapidly injected into each patient via the left elbow vein, followed by rapid injection of $5 \mathrm{ml}$ saline. The contrast mode was applied and the dynamic development process of the breast lesion was observed for 3-5 min. The dynamic imaging acquisition time was $>3 \mathrm{~min}$. The morphology of the lesion, time to initiation of enhancement, time to peak (TTP) and peak intensity of the TIC were analyzed.

Evaluation criteria. The lesions' morphology on contrastenhanced US and perfusion pattern were assessed, with the major features observed including whether the lesion shape was regular, whether the boundary was clear and the distorted vascular shape, as well as contrast agent distribution, filling defects and wash-out mode. The enhancement morphology of each mass was classified, with the major categories including scattered punctuate enhancement, circular or semi-circular enhancement, dendritic enhancement, partial enhancement and overall enhancement. The surrounding tissue was set to moderate enhancement and the enhancement pattern at different levels during different time-periods was recorded and analyzed.

Image analysis. The perfusion processes of breast tissue and lesions was reviewed and analyzed with the in-built acoustic TIC analysis software (EsaoteSpA). The region of interest of the breast lesion and surrounding tissue was manually determined and the TIC was obtained and analyzed. TICs for malignant and benign breast lesions were generated and analyzed using the Wash-in/Wash-out software (Qlab HC795041VS; Philips Medical Systems, Inc.).
Statistical analysis. Values are expressed as the mean \pm standard deviation. SPSS 17.0 software (SPSS, Inc.) was used for statistical analysis. Quantitative data conformed to the normal distribution. Comparisons were performed using analysis of variance, the Student's t-test or the $\mathrm{c} 2$ test. The area under the curve (AUC), as well as the sensitivity and specificity of the ROC were analyzed. $\mathrm{P}<0.05$ was considered to indicate a statistically significant difference.

\section{Results}

Imaging features of contrast-enhanced ultrasound. The imaging features of contrast-enhanced ultrasound of malignant and benign breast lesions were analyzed. For the malignant breast tumors, typical features included irregular shape and vascular morphology, uneven contrast agent distribution, filling defects and contrast agent retention, 'fast-out' wash-out mode, unclear boundaries and uneven internal echo. Conversely, benign lesions were characterized by 'slow-out' or synchronous wash-out mode. The results indicated obvious differences in the imaging features of contrast-enhanced ultrasound between the malignant and benign lesion groups $(\mathrm{P}<0.05$; Table I).

TIC analysis of malignant and benign breast lesions. Parameters of hemodynamics and perfusion for the malignant and benign breast lesions were next investigated. The results indicated that, compared with the benign lesions, the perfusion time and TTP were significantly earlier for the malignant lesions $(\mathrm{P}<0.05)$. However, the wash-out time for the malignant breast lesions was later than that for the benign breast lesions. Furthermore, compared with those of the benign breast lesions, a significantly greater PI, rising slope and AUC were observed for the malignant breast lesions $(\mathrm{P}<0.05$; Table II).

Perfusion performance of malignant and benign breast lesions. A significantly different enhancement performance was observed during different time-periods (Fig. 1 and 2). For the malignant breast lesions, uneven partial enhancement and overall enhancement were mainly observed. Conversely, the benign breast lesions were mainly characterized by local punctuate enhancement, circular enhancement and linear enhancement. Furthermore, in contrast to the findings based on angiography, all of the malignant breast lesions exhibited an enlarged focus scope. The breast cancer group comprised 42 cases with high enhancement, 7 cases with moderate enhancement and 3 cases with low enhancement. However, the focus scope enhancement was not obvious for the benign breast lesions, comprising 25 cases of high enhancement, 13 cases of moderate enhancement, 29 cases of low enhancement and 6 cases of no enhancement.

TIC morphology analysis of malignant and benign breast lesions. TICs for malignant and benign breast lesions indicated that $88.4 \%$ of the TICs for the malignant breast lesions were of the fast-rising and slow-declining type, while 75.3 and $17.8 \%$ of the TICs for the benign breast lesions were of the slow-rising and fast-declining and of the fast-rising and fast-declining type, respectively. Significant differences in the TIC shapes were observed between the malignant and benign breast lesion groups $(\mathrm{P}<0.05$; Table III; Fig. 3). 
Table I. Contrast-enhanced ultrasound characteristics of benign and malignant breast lesions.

\begin{tabular}{|c|c|c|c|c|}
\hline Feature & Benign lesions $(n=73)$ & Malignant lesions $(\mathrm{n}=52)$ & $\chi^{2}$ & $\mathrm{P}$-value \\
\hline \multicolumn{5}{|l|}{ Morphology } \\
\hline Irregular & $24(32.8)$ & $38(73.1)$ & 19.6 & 0.0002 \\
\hline Regular & $49(76.2)$ & $14(26.9)$ & & \\
\hline \multicolumn{5}{|l|}{ Boundary } \\
\hline Unclear & $15(20.5)$ & $32(61.5)$ & 21.7 & 0.0007 \\
\hline Clear & $58(79.4)$ & $20(38.4)$ & & \\
\hline \multicolumn{5}{|l|}{ Vascular morphology } \\
\hline Irregular & $29(39.7)$ & $43(82.6)$ & 22.9 & $<0.0001$ \\
\hline Regular & $44(60.2)$ & $9(17.3)$ & & \\
\hline \multicolumn{5}{|l|}{ Contrast agent distribution } \\
\hline Uneven & $11(15.0)$ & $40(76.9)$ & 48.1 & 0.0005 \\
\hline Even & $62(84.9)$ & $12(23.1)$ & & \\
\hline \multicolumn{5}{|l|}{ Filling defects } \\
\hline Yes & $13(17.8)$ & $46(88.4)$ & 60.8 & 0.0002 \\
\hline No & $60(82.1)$ & $6(11.6)$ & & \\
\hline \multicolumn{5}{|l|}{ Wash-out retention } \\
\hline Yes & $18(24.6)$ & $48(92.3)$ & 55.7 & 0.0001 \\
\hline No & $55(75.3)$ & $4(7.7)$ & & \\
\hline \multicolumn{5}{|l|}{ Wash-out mode } \\
\hline Fast-out & $8(10.9)$ & $35(67.3)$ & 42.7 & 0.0005 \\
\hline Slow-out or synchronous & $65(89.1)$ & $17(32.7)$ & & \\
\hline
\end{tabular}

Values are expressed as $\mathrm{n}(\%)$.

Table II. Perfusion parameter analysis of malignant and benign breast lesions.

\begin{tabular}{lccr}
\hline Parameter & Benign $(\mathrm{n}=73)$ & Malignant $(\mathrm{n}=52)$ & P-value \\
\hline Perfusion time $(\mathrm{sec})$ & $10.34 \pm 1.96$ & $5.96 \pm 1.47$ & 0.01 \\
Perfusion intensity $\left(\mathrm{e}^{-0.02}\right)$ & $4.15 \pm 2.03$ & $2.83 \pm 1.46$ & 0.02 \\
Time to peak (sec) & $26.73 \pm 7.26$ & $16.38 \pm 3.58$ & $<0.01$ \\
Peak intensity (e-0.02) & $6.73 \pm 4.21$ & $10.42 \pm 5.47$ & 0.03 \\
Wash-out time (sec) & $103.34 \pm 24.67$ & $147.72 \pm 30.26$ & 0.01 \\
Rising slope $(1 / \mathrm{sec})$ & $3.74 \pm 2.17$ & $9.42 \pm 3.74$ & 0.01 \\
Declining slope $(1 / \mathrm{sec})$ & $5.04 \pm 2.62$ & $4.24 \pm 1.69$ & 0.04 \\
\hline
\end{tabular}

Values are expressed as the mean \pm standard deviation. The perfusion time refers to the duration covering the period from the contrast agent entering the lesion (after the enhanced arterial phase) until the time that there was no significant difference between the lesion and surrounding normal tissues (after the vein phase).

ROC curve analysis of TIC parameters. ROC curves were constructed based on the TIC parameters, including peak intensity, TTP, wash-out time and rising slope. According to the best threshold principle for ROC analysis (10), the point with the greatest Youden index (sensitivity + specificity - 1) was considered as the threshold, i.e., a TTP of $\leq 14.43 \mathrm{sec}$, wash-out time of $\geq 149.76 \mathrm{sec}$ and rising slope of $\geq 10.551 / \mathrm{sec}$. The sensitivity and specificity of the various parameters for the diagnosis of malignant breast lesions are presented in Table IV. The results suggested that the sensitivity of the wash-out time was slightly but not significantly lower than that of the TTP and the rising slope $(\mathrm{P}>0.05)$. The AUC values for the peak intensity, TTP, wash-out time and rising slope for the diagnosis of malignant breast lesions were 0.696, 0.870,0.841 and 0.816, respectively. The results indicated that the TTP, wash-out time and rising slope have a diagnostic value for malignant breast lesions (Fig. 4). 
Table III. Time-intensity curve shape analysis of malignant and benign breast lesions.

\begin{tabular}{lccr}
\hline Parameter & Benign $(\mathrm{n}=73)$ & Malignant $(\mathrm{n}=52)$ & P-value \\
\hline Fast-rising and slow-declining & $5(6.8)$ & $46(88.4)$ & $<0.01$ \\
Slow-rising and fast-declining & $55(75.3)$ & $4(7.6)$ & $<0.01$ \\
Fast-rising and fast-declining & $13(17.8)$ & $2(3.8)$ & 0.01 \\
\hline
\end{tabular}

Values are expressed as $\mathrm{n}(\%)$.

A

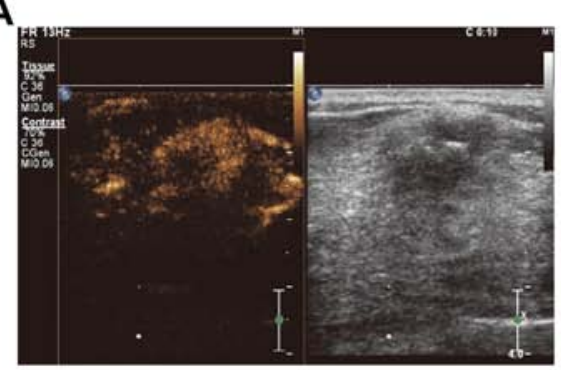

B

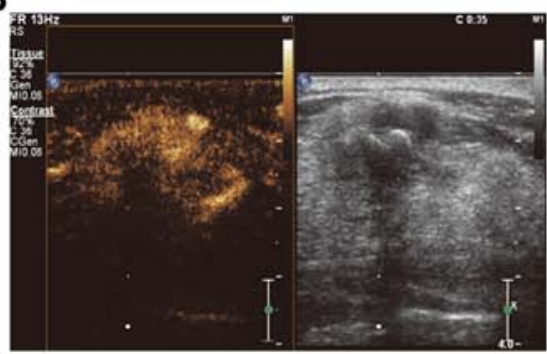

C

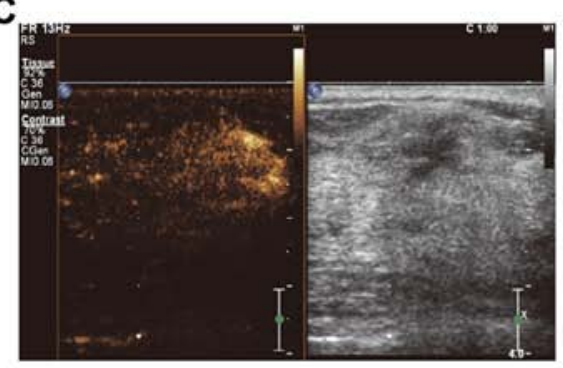

Figure 1. Contrast-enhanced ultrasound performance in a patient with malignant breast lesions. (A) 10 sec: Uneven influx of contrast agent with partial enhancement. (B) $35 \mathrm{sec}$ : Rich influx of contrast agent with overall enhancement. (C) 60 sec: Slow decline of contrast agent within malignant lesions. In the two-dimensional black/white image, the black signal indicated the presence of the lesion.

A

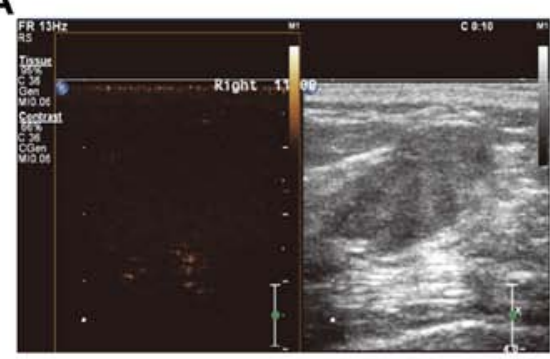

B

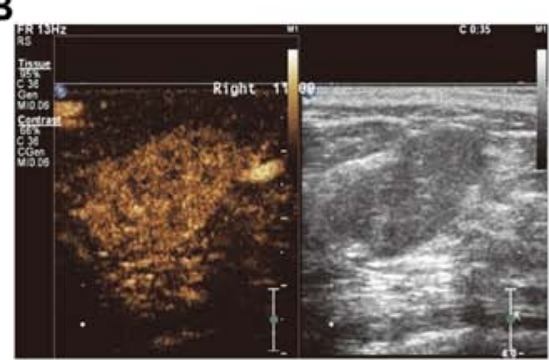

C

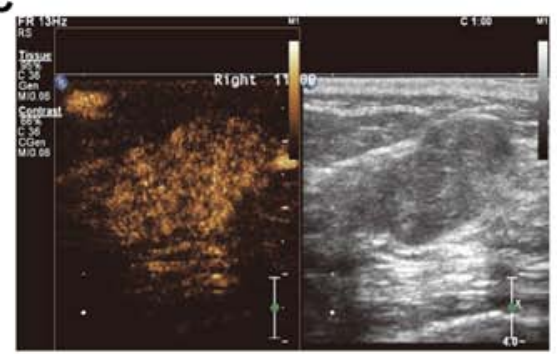

Figure 2.Contrast-enhanced ultrasound performance in a patient with benign breast lesions. (A) 10 sec: Relatively less influx of contrast agent, with punctuate enhancement. (B) $35 \mathrm{sec}$ : Circular enhancement within the benign lesion. (C) $60 \mathrm{sec}$ : Multiple linear enhancement within the lesion, with fast decline of contrast agent. In the colored image, stronger signals indicated lesions. In the two-dimensional black/white image, the black signal indicated the presence of the lesion.

\section{Discussion}

Breast cancers rank first among malignancies in females and clinical symptoms are mild at the early stage (11). At the time-point of diagnosis, breast cancer may have progressed to the intermediate or late stage, which is associated with high mortality. Therefore, early disease detection and diagnosis may provide a marked benefit for breast cancer treatment and prognosis, as well as the survival rate and quality of life of affected patients (12). At present, the diagnosis of breast cancer mainly depends on imaging methods. Along with the rapid development of imaging technologies in recent years, the ability of clinicians to differentiate between malignant and benign breast lesions has markedly improved (13).

The new-generation contrast agent SonoVue is able to clearly visualize the microcirculation of the breast lesion (14), making true microcirculation imaging possible (15). Microcirculation imaging, performed as an additional round of imaging following the conventional ultrasound, is able to reflect the blood flow perfusion of normally structured and tumor tissue (16), under color Doppler and energy Doppler flow imaging. To date, clinical studies have indicated that application of contrast agents significantly improves the diagnostic accuracy for breast lesions. Contrast-enhanced ultrasound is able to display the microvascular structure in the diameter range of 20-39 $\mu \mathrm{m}$ (17), and is a reliable method for the evaluation of the tumor microvascular circulation. Kettenbach et al (18), indicated that detection of the vascular morphology by the computer-assisted quantitative assessment of power Doppler US might be one of the most suitable methods for distinguishing between malignant and benign breast lesions.

In the present study, the breast lesions whose diagnosis was not possible by conventional ultrasound were subjected to contrast-enhanced ultrasound. The enhancement morphology and perfusion pattern of the breast lesions were analyzed, and the clinical application value of contrast-enhanced ultrasound was investigated. All of the included subjects were finally 
Table IV. Sensitivity and specificity analysis of TIC perfusion parameters of malignant and benign breast lesions.

\begin{tabular}{lcccr}
\hline TIC parameter & Sensitivity $(\%)$ & 1-specifity $(\%)$ & Youden index & AUC \\
\hline Time to peak & 89.7 & 17.4 & 14.43 & 0.870 \\
Wash-out time & 85.6 & 14.3 & 149.76 & 0.841 \\
Rising slope & 72.4 & 26.1 & 10.55 & 0.816 \\
\hline
\end{tabular}

TIC, time-intensity curve; AUC, area under the curve.

A

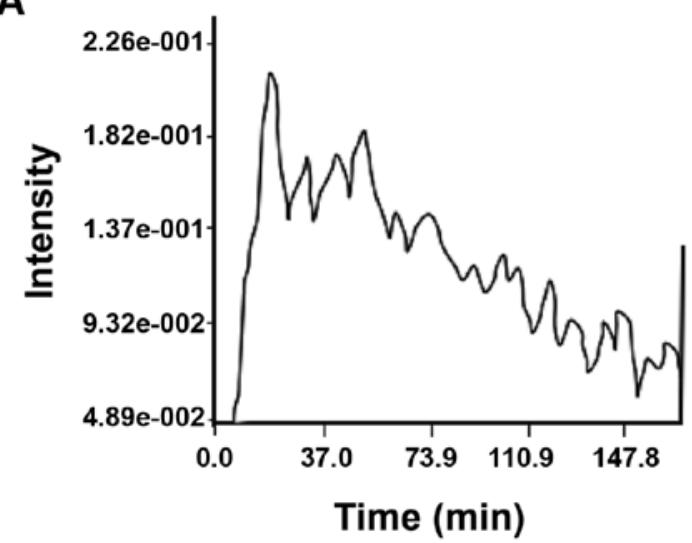

B

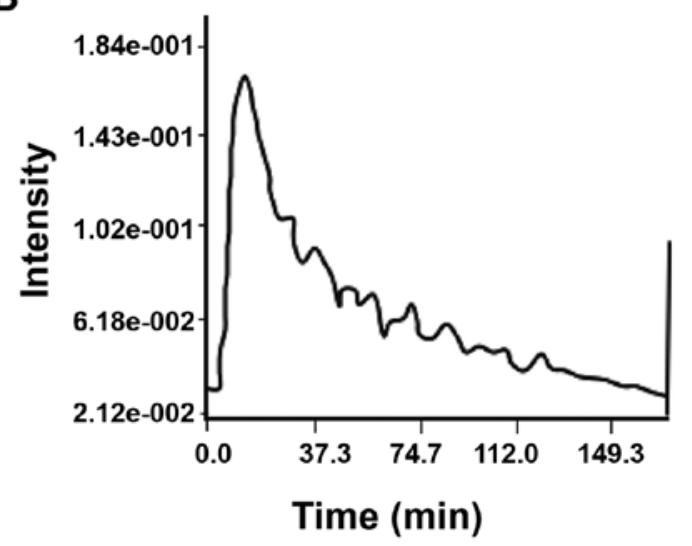

Figure 3. TIC morphology analysis of malignant and benign breast lesions. (A) Representative TIC for malignant breast lesions, exhibiting a fast-rising and slow-declining platform shape. (B) Representative TIC for benign breast lesions of the slow-rising type. TIC, time-intensity curves.

diagnosed by pathology. The focal lesion morphology, lesion boundaries, vascular morphology within lesions, contrast agent distribution, wash-out mode and focal range of these malignant and benign breast lesions were observed by contrast-enhanced ultrasound detection. In a previous study, irregular radial boundaries were observed on $84.6 \%$ of the malignant breast lesions on contrast-enhanced ultrasound, while mass lumps were observed for benign breast lesions, and only $29.4 \%$ of the benign breast lesions had a regular morphology (19). In a study by Zhao et al (20), 91.1\% of malignant breast lesions exhibited a crab claw-like enhancement, while $83.9 \%$ of the benign breast lesions were homogeneously enhanced. In the present study, malignant breast lesions were mainly characterized by an irregular appearance and vascular morphology on contrast-enhanced ultrasound detection, and an uneven contrast agent distribution with filling defects and contrast agent retention was observed. Furthermore, the malignant breast lesions mainly exhibited uneven partial enhancement and overall enhancement, with the TICs being of the fastrising and slow-declining type, and the fast-out wash-out mode, as well as unclear boundaries and uneven echo were common. On the contrary, the benign breast lesions mainly exhibited local punctuate enhancement, circular enhancement and linear enhancement, and TICs were of the slow-rising and fast-declining, and fast-rising and fast-declining types, and the slow-out or synchronous wash-out modes were mainly observed.

The TIC represents the dynamic process of contrastenhanced ultrasound, which accurately reflects the differential

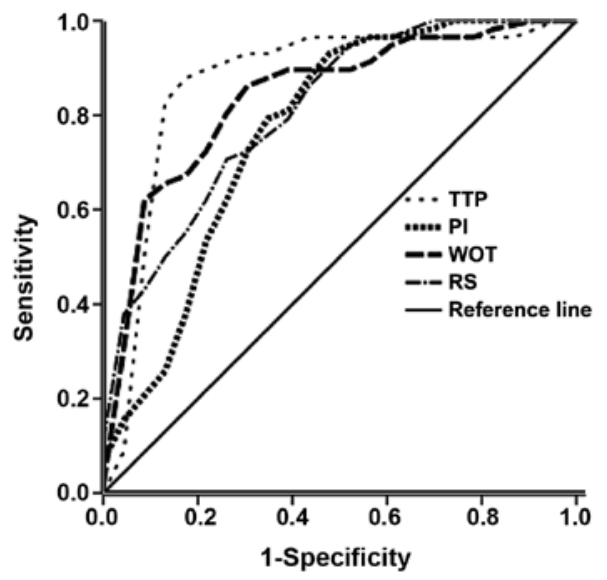

Figure 4.Receiver operating characteristics analysis of the time-intensity curve parameters. PI, contrast agent perfusion starting time/intensity; TTP, time to peak intensity; WOT, wash-out time; RS, rising slope.

blood perfusion and wash-out processes of malignant and benign breast lesions. In the present study, TIC analysis suggested that $88.4 \%$ of the TICs of the malignant breast lesions were of the fast-rising and slow-declining type, which may be mainly attributed to angiogenesis and abnormal blood supply within tumors. Abnormal blood vessels are prone to venous thrombus, which may interfere with the blood supply and lead to contrast agent retention. Furthermore, the TICs of $75.3 \%$ of the benign breast lesion were of the slow-rising 
and fast-declining type, while $17.8 \%$ were of the fast-rising and fast-declining type. This phenomenon may be due to the fact that angiogenesis within benign breast lesions may result from normal blood vessels, or their hyperplasia and thickening. There was no abnormal vascular network or arteriovenous fistula within the benign lesions, and the normal venous drainage did not result in any contrast agent retention. In the ROC analysis, the AUC values for the peak intensity, time to peak, wash-out time and rising slope were 0.696 , $0.870,0.841$ and 0.816 , respectively, indicating a satisfactory clinical value for the time to peak, wash-out time and rising slope in diagnosing malignant breast lesions. These results are in accordance with those of a previous study (21). These phenomena may be explained by the notion that, due to the rich angiogenesis and blood flow perfusion within masses, the perfusion parameters reflect the top transient amount of microbubbles and the flow of contrast agent during a certain period. Regarding the sensitivity and specificity analysis, the Youden indexes for time to peak, wash-out time and rising slope were $14.43,149.76$ and 10.55 , respectively, while the sensitivities were 89.7, 85.6 and 72.4, respectively. As a tool for authenticity assessment, changes in the Youden index may provide imaging information for distinguishing between malignant and benign breast lesions in the clinic. Of note, all of the three parameters had high sensitivities for diagnosing malignant breast lesions, indicating that these three indicators may reflect the angiogenesis within the breast masses. However, the present study was only a preliminary investigation of the diagnostic value of perfusion parameters from contrast-enhanced ultrasound. Further in-depth studies with optimized diagnostic indicators and larger sample sizes are still required in the future.

In conclusion, the present study indicated that the TIC parameters from contrast-enhanced ultrasound of breast lesions have promising clinical value in differentiating between malignant and benign lesions. Time to peak, washout time and rising slope may contribute to the identification of malignant breast lesions, which may facilitate early treatment and patient prognosis.

\section{Acknowledgements}

Not applicable.

\section{Funding}

This study was supported by the Science and Technology Benefit Project (grant no. 2017c50070).

\section{Availability of data and materials}

The datasets used and/or analyzed during the present study are available from the corresponding author on reasonable request.

\section{Authors' contributions}

$\mathrm{YZ}, \mathrm{MZ}, \mathrm{XF}$ and DM contributed to the study design, experimental performance, data collection/analysis, and manuscript preparation. All authors read and approved the final manuscript.

\section{Ethical approval and consent to participate}

Written informed consent was obtained from each patient and the study was approved by the Ethics Review Board of the Ningbo No. 2 Hospital (Ningbo, Zhejiang, China).

\section{Patient consent for publication}

Not applicable

\section{Competing interests}

All authors declare that they have no competing interests.

\section{References}

1. Grayson M: Breast cancer. Nature 485: S49, 2012.

2. Johnson RH, Hu P, Fan C and Anders CK: Gene expression in 'young adult' type breast cancer: A retrospective analysis. Oncotarget 6: 13688-13702, 2015.

3. Howell A, Anderson AS, Clarke RB, Duffy SW, Evans DG, Garcia-Closas M, Gescher AJ, Key TJ, Saxton JM and Harvie MN: Risk determination and prevention of breast cancer. Breast Cancer Res 16: 446, 2014.

4. Watanabe T, Kaoku S, Yamaguchi T, Izumori A, Konno S, Okuno T, Tsunoda H, Ban K, et al: Multicenter Prospective Study of Color Doppler Ultrasound for Breast Masses: Utility of Our Color Doppler Method. Ultrasound Med Biol 45: 1367-1379, 2019.

5. Purushothaman HN, Lekanidi K, Shousha S and Wilson R: Lesions of uncertain malignant potential in the breast (B3): What do we know? Clin Radiol 71: 134-140, 2016.

6. JGuo R and Lu G: Ultrasound Imaging Technologies for Breast Cancer Detection and Management: A Review. Ultrasound Med Biol 44: 37-70, 2018.

7. Wang YM, Fan W, Zhang K, Zhang L, Tan Z and Ma R: Comparison of transducers with different frequencies in breast contrast-enhanced ultrasound (CEUS) using SonoVue as contrast agent. Br J Radiol 89: 20151050, 2016.

8. Sadeghi-Naini A, Sannachi L, Pritchard K, Trudeau M, Gandhi S, Wright FC, Zubovits J, Yaffe MJ, Kolios MC and Czarnota GJ: Early prediction of therapy responses and outcomes in breast cancer patients using quantitative ultrasound spectral texture. Oncotarget 5: 3497-3511, 2014.

9. Liu J, Wang D, Li H, Li H, Zhou T, Zhao S and Ding Z: Clinical value of contrast-enhanced ultrasound in diagnosis of hyperechoic liver lesions. Med Sci Monit 21: 2845-2850, 2015.

10. Obuchowski NA amd Bullen JA: Receiver operating characteristic (ROC) curves: review of methods with applications in diagnostic medicine. Phys Med Biol 63: 07TR01, 2018.

11. Johnson CJ, Graff R, Moran P, Cariou C and Bordeaux S: Breast cancer stage, surgery, and survival statistics for Idaho's National Breast and Cervical Cancer Early Detection Program population, 2004-2012. Prev Chronic Dis 12: E36, 2015.

12. Cance WG, Carey LA, Calvo BF, Sartor C, Sawyer L, Moore DT, Rosenman J, Ollila DW and Graham M II: Long-term outcome of neoadjuvant therapy for locally advanced breast carcinoma: Effective clinical downstaging allows breast preservation and predicts outstanding local control and survival. Ann Surg 236: 295-302, discussion 302-303, 2002.

13. Alunni-Fabbroni M, Müller V, Fehm T, Janni W and Rack B: Monitoring in metastatic breast cancer: Is imaging outdated in the era of circulating tumor cells? Breast Care (Basel) 9: 16-21, 2014.

14. Xiao X, Ou B, Yang $\mathrm{H}, \mathrm{Wu} \mathrm{H}$ and Luo B: Breast contrastenhanced ultrasound: Is a scoring system feasible? A preliminary study in China. PLoS One 9: e105517, 2014.

15. Madjar H, Prömpeler HJ, Del Favero C, Hackelöer BJ and Llull JB: A new Doppler signal enhancing agent for flow assessment in breast lesions. Eur J Ultrasound 12: 123-130, 2000.

16. Wang J, Qin B, Chen X, Wagner WR and and Villanueva FS: Ultrasound mol imaging angiogenesis using vasc endothelial growth factor-conjugated microbubbles. Mol Pharmaceutics 14: 781-790, 2017. 
17. Forsberg F, Kuruvilla B, Pascua MB, Chaudhari MH, Merton DA Palazzo JP and Goldberg BB: Comparing contrast-enhanced color flow imaging and pathological measures of breast lesion vascularity. Ultrasound Med Biol 34: 1365-1372, 2008.

18. Kettenbach J, Helbich TH, Huber S, Zuna I and Dock W: Computer-assisted quantitative assessment of power Doppler US: Effects of microbubble contrast agent in the differentiation of breast tumors. Eur J Radiol 53: 238-244, 2005.

19. Gity M, Arabkheradmand A, Taheri E and Shakiba M: Diagnostic investigation of breast magnetic resonance imaging in malignant and benign mass lesions. Arch Med Sci 14: 1061-1069, 2018.
20. Zhao H, Xu R, Ouyang Q, Chen L, Dong B and Huihua Y: Contrastenhanced ultrasound is helpful in the differentiation of malignant and benign breast lesions. Eur J Radiol 73: 288-293, 2010

21. Amioka A, Masumoto N, Gouda N, Kajitani K, Shigematsu H, Emi A, Kadoya T and Okada M: Ability of contrast-enhanced ultrasonography to determine clinical responses of breast cancer to neoadjuvant chemotherapy. Jpn J Clin Oncol 46: 303-309, 2016.

c) (i) () $($ This work is licensed under a Creative Commons COY AC Attribution-NonCommercial-NoDerivatives 4.0 International (CC BY-NC-ND 4.0) License. 